\title{
Plasticity in high-order cognition: Evidence of dissociation in aphasia
}

DOI: $10.1017 /$ S0140525X07001318

Rosemary Varley

Human Communication Sciences, University of Sheffield, Sheffield S10 2TA, United Kingdom.

r.a.varley@sheffield.ac.uk http://www.shef.ac.uk/hcs/staff/varley

Abstract: High-order constructs such as intelligence result from the interaction of numerous processing systems, one of which is language. However, in determining the role of language in intelligence, attention must be paid to evidence from lesion studies and, in particular, evidence of dissociation of functions where high-order cognition can be demonstrated in face of profound aphasia.

Jung \& Haier (J\&H) present a broad-ranging review of research into the neural bases of intelligence. They synthesize evidence from functional neuroimaging, genetic, and lesion studies. The neuroimaging and lesion research appear to converge on the notion that language and the neural systems that underpin it are an essential component of intelligence. Included in the P-FIT network are left hemisphere Brodmann Areas (BAs) 22, $39,40,44$, and 45 , all of which are central to language function and lesions of which result in aphasia. J\&H review mid-twentieth-century research for evidence of decline of intelligence in aphasia. They identify the common theme that people with Broca's aphasia, and lesions in and around BAs 44 and 45, can show no reduction in performance on nonverbal intelligence scales.

This conclusion is supported by more recent research showing that people with severe agrammatic aphasia, and also marked lexical impairment, can still sustain high-level intellectual performances in domains including social, causal, and mathematical reasoning (Varley 2002; Varley \& Siegal 2000; Varley et al. 2005). The group of aphasic patients for which there are consistent reports of cognitive decline are those with global aphasia (e.g., van Mourik et al. 1992). However, individuals with global aphasia often have very large left hemisphere lesions, spanning much of J\&H's P-FIT network, and it becomes difficult to determine whether cognitive decline in these cases is due to impairment of language or to loss of some other cognitive subsystem that is geographically close to, or overlapping with, the language network. In addition, the extent of disconnection of functional systems that occurs with large lesions might account for cognitive decline, independent of a specific role for language in high-level cognition.

The evidence that some individuals with lesions within the P-FIT network can still sustain high-order intellectual activities presents an apparent contradiction to the results of functional imaging studies that show recruitment of the same zones in a range of intellectual tasks. The behavioral tasks used to 
investigate intelligence are varied, but they all represent highdemand cognitive activities. Typical tasks might involve rapid perceptual-motor recoding of information, loading of working memory, deductive and inductive reasoning, and the frequent formation and shifting of strategies. Successful completion of such tasks typically involves the recruitment of a bundle of cognitive mechanisms. Some of these mechanisms will be core to the completion of the task (such as visual perceptual processes in a matrix reasoning test), whereas others represent more fluid "co-opted" mechanisms that scaffold performance (Clark 1998; Siegal \& Varley 2002).

The status of language as a "core" versus "co-opted" mechanism is crucial in many debates as to the role of language in thought and high-order cognition. There are aspects of language which make it an excellent candidate for core status in many forms of reasoning. The lexicon provides a set of symbols that permits the encapsulation and manipulation of abstract notions such as spatial relationships or large numerosities (Dehaene et al. 1999; Hermer-Vasquez et al. 1999). Similarly, the grammatical mechanisms of language might be crucial, permitting the capture of relationships between entities. However, the language faculty is also a prime candidate for co-opted status. Language, either in the form of overt verbalization, or as covert inner speech, may appear in many tasks, including ones that involve the manipulation of abstract visuo-spatial information, such as matrix reasoning tests. The breaking down of a complex problem into a series of sub-steps represented in language sentences may represent an important cognitive tool in solving that problem. Similarly, the encoding of information into phonological form permits rehearsal and maintenance in working memory of intermediate products of problem solving.

Functional brain imaging studies allow identification of the neural mechanisms that are associated with the performance of a particular cognitive task. Hence, activation of left hemisphere peri-sylvian zones might indicate that language is a component of the activity under investigation. However, functional imaging data cannot discriminate whether language-related activation corresponds to a core or co-opted component of an activity. To some degree, appropriate linguistic baseline conditions in an imaging study might clarify this issue, and linguistic processing during baseline scanning might permit the subtraction of generalized activity from activity in experimental conditions. However, it is not clear that the language demands of a control condition necessarily equate to those of the active internal dialogue that is ubiquitous in high-demand cognitive activities. Passively viewing sentences or making decisions as to whether firstmentioned nouns are human agents (Goel et al. 1997) does not necessarily match the functional demands of forming a series of natural language sentences during problem solving.

J\&H's scholarly synthesis of evidence from diverse domains is to be welcomed. However, in reviewing neuropsychological studies it is important to consider the evidence of dissociation of functions and that high-order cognition can be retained despite large lesions within the P-FIT network. Such evidence suggests flexibility and plasticity in the mechanisms that are recruited to perform a task. The neurobiological substrate of phonological working memory lies within the P-FIT network. However, when the capacity to encode and maintain information in phonological form is impaired, as it invariably is in aphasia, alternative resources such as visuo-spatial working memory can be recruited in order to sustain a performance. Many of the regions identified by $\mathrm{J} \& \mathrm{H}$ may reflect the mechanisms that are ordinarily recruited by healthy participants to complete a demanding intellectual task. For most human participants, language represents a fine resource to support thinking and reasoning. However, the evidence from people with severe aphasia suggests that these regions and the functions that they sustain are not necessary for high-order cognition. Intelligence may be characterized both at the behavioural and neural levels by flexibility and plasticity. 